\title{
Korn inequalities for shells with zero Gaussian curvature
}

\author{
Yury Grabovsky* Davit Harutyunyan ${ }^{\dagger}$
}

September 4, 2018

\begin{abstract}
We consider shells with zero Gaussian curvature, namely shells with one principal curvature zero and the other one having a constant sign. Our particular interests are shells that are diffeomorphic to a circular cylindrical shell with zero principal longitudinal curvature and positive circumferential curvature, including, for example, cylindrical and conical shells with arbitrary convex cross sections. We prove that the best constant in the first Korn inequality scales like thickness to the power $3 / 2$ for a wide range of boundary conditions at the thin edges of the shell. Our methodology is to prove, for each of the three mutually orthogonal two-dimensional cross-sections of the shell, a "first-and-a-half Korn inequality" - a hybrid between the classical first and second Korn inequalities. These three two-dimensional inequalities assemble into a three-dimensional one, which, in turn, implies the asymptotically sharp first Korn inequality for the shell. This work is a part of mathematically rigorous analysis of extreme sensitivity of the buckling load of axially compressed cylindrical shells to shape imperfections.
\end{abstract}

\section{Introduction}

Classical first and second Korn inequalities have been known to play a central role in the theory of linear elasticity and recently they have found very important applications in the problems of buckling of slender structures [6, 4, 5]. Let us recall the classical first and second Kohn inequalities, that actually date back to 1908, [8, 9]. To that end we denote

$$
\mathfrak{e u c}(n)=\left\{\boldsymbol{u}: \mathbb{R}^{n} \rightarrow \mathbb{R}^{n}: \boldsymbol{u}(\boldsymbol{x})=\boldsymbol{A} \boldsymbol{x}+\boldsymbol{b}, \boldsymbol{A} \in \operatorname{Skew}\left(\mathbb{R}^{n}\right), \boldsymbol{b} \in \mathbb{R}^{n}\right\}
$$

be the set of all infinitesimal motions, i.e., a Lie algebra of the group of all Euclidean transformations (rigid body motions). Let $\Omega$ be an open connected subset of $\mathbb{R}^{n}$ and $\boldsymbol{u} \in$ $W^{1,2}\left(\Omega ; \mathbb{R}^{n}\right)$. We denote 1 by $\operatorname{grad} \boldsymbol{u}$ and $(\operatorname{grad} \boldsymbol{u})_{\text {sym }}$ the gradient and the symmetric part

*Temple University, Philadelphia, PA, yury@temple.edu

†University of Utah, Salt Lake City, UT, davith@math.utah.edu

${ }^{1}$ We reserve more streamlined notations $\nabla \boldsymbol{u}$ and $e(\boldsymbol{u})$ for "simplified" gradient and symmetrized gradient, respectively, that will be our main characters in the technical part of the paper. 
of the gradient, respectively, of a vector field $\boldsymbol{u}$. It is well-known that $(\operatorname{grad} \boldsymbol{u})_{\mathrm{sym}}=0$ in $\Omega$ (in the sense of distributions) if and only if $\boldsymbol{u} \in \mathfrak{e u c}(n)$. This is an immediate consequence of a simple observation (also very well-known) that all partial derivatives of the gradient $\boldsymbol{G}=\operatorname{grad} \boldsymbol{u}$ can be expressed as a linear combination of partial derivatives of the symmetric part of the gradient $\boldsymbol{E}=(\operatorname{grad} \boldsymbol{u})_{\mathrm{sym}}$ :

$$
\frac{\partial G_{j k}}{\partial x^{i}}=\frac{\partial E_{j k}}{\partial x^{i}}+\frac{\partial E_{i j}}{\partial x^{k}}-\frac{\partial E_{i k}}{\partial x^{j}} .
$$

The classical first Korn inequality (e.g., as stated in [14]) quantifies this result by describing how large $(\operatorname{grad} \boldsymbol{u})_{\text {sym }}$ must be if $\boldsymbol{u}$ lies in a closed subspace $V \subset W^{1,2}\left(\Omega ; \mathbb{R}^{n}\right)$ that has trivial intersection with $\mathfrak{e} \mathfrak{u}(n)$. If $\Omega$ is a Lipschitz domain, then there exists a constant $C(\Omega, V)$, such that for every $\boldsymbol{u} \in V$

$$
\left\|(\operatorname{grad} \boldsymbol{u})_{\mathrm{sym}}\right\|^{2} \geq C(\Omega, V)\|\operatorname{grad} \boldsymbol{u}\|^{2},
$$

where $\|\cdot\|$ is the $L^{2}$-norm. The Korn constant $C(\Omega, V)$ measures the distance between the subspace $V$ and $\mathfrak{e u c}(n)$. The classical second Korn inequality asserts that the standard $W^{1,2}$ norm topology can be equivalently defined by replacing grad $\boldsymbol{u}$ with $(\operatorname{grad} \boldsymbol{u})_{\text {sym }}$ :

$$
\|\operatorname{grad} \boldsymbol{u}\|^{2} \leq C(\Omega)\left(\left\|(\operatorname{grad} \boldsymbol{u})_{\mathrm{sym}}\right\|^{2}+\|\boldsymbol{u}\|^{2}\right), \quad \boldsymbol{u} \in W^{1,2}\left(\Omega ; \mathbb{R}^{n}\right) .
$$

Originally, Korn inequalities were used to prove existence, uniqueness and well-posedness of boundary value problems of linear elasticity (see e.g., [11, 1]). Nowadays, often, as in our particular case, it is the best Korn constant $C(\Omega, V)$ in the first Korn inequality that is of central importance (e.g., [2, 12, 13, 15, 16, 10]). Specifically, we are interested in the asymptotic behavior of the Korn constant $K\left(\Omega_{h}, V_{h}\right)$ for shells with zero Gaussian curvature as a function of their thickness $h$ for subspaces $V_{h}$ of $W^{1,2}$ functions satisfying various boundary conditions at the thin edges of the shell. In [6, 5] we have shown that $K\left(\Omega_{h}, V_{h}\right)$ represents an absolute lower bound on safe loads for any slender structure. For a classical circular cylindrical shell we have proved in [3] that $K\left(\Omega_{h}, V_{h}\right) \sim h^{3 / 2}$ for a broad class of boundary conditions at the thin edges of the shell.

The motivation for this work comes from the fact that the experimentally measured buckling loads of axially compressed cylindrical shells behave in a paradoxical way, dramatically disagreeing with predictions of classical shell theory. The universal consensus is that such behavior is due to the extreme sensitivity of shells to imperfections of shape and load. This study is a part of rigorous analytical investigation of the influence of small changes in shape on the structural behavior of cylindrically-shaped shells. It looks like (and this will be addressed in future work) the determining factor of the effect of shape imperfections is the Gaussian curvature of the actual imperfect shell as the Ansatzen suggest in [17]. In this paper we show that if the shell has a vanishing principal curvature (yielding zero Gaussian curvature), like circular cylindrical shells, then the scaling of the Korn constant $K\left(\Omega_{h}, V_{h}\right)$ will remain unaffected, provided the non-zero principal curvature has a constant sign. Our analysis also shows that if both principal curvatures are zero on any open subset of the shell's mid-surface, then $K\left(\Omega_{h}, V_{h}\right) \sim h^{2}$. We conjecture that $K\left(\Omega_{h}, V_{h}\right) \sim h$ for shells of uniformly positive Gaussian curvature, while $K\left(\Omega_{h}, V_{h}\right) \sim h^{4 / 3}$ if the Gaussian curvature is negative 
on any open subset of the shell's middle surface, as suggested by test functions constructed in [17]. These conjectures will be addressed elsewhere.

The goal of this paper is to show that the tools developed in [3] for circular cylindrical shells, and extended and developed further in [7], possess enough flexibility to be applicable to a wide family of shells and in particular cylindrically-shaped shells (the ones, that have no boundary in one of the principal directions). The main idea is to first prove an inequality that is a hybrid between the first and second Korn inequalities (we call it "first-and-ahalf Korn inequality" for this reason) by "assembling" it from its two-dimensional versions corresponding to cross-sections of the shell by curvilinear coordinate surfaces. The first Korn inequality is then a consequence of the first-and-a-half Korn inequality and an estimate on the normal component of $\boldsymbol{u} \in V_{h}$. We believe that this general methodology will work for broad classes of shells, even though the Gaussian curvature does affect the validity of some of the technical steps in the proof, which must be adjusted to the particular case. In particular, the assumption of zero Gaussian curvature is essential for all main results in Section 3 .

\section{Preliminaries}

Consider a shell whose mid-surface is of class $C^{2}$. Suppose $z$ and $\theta$ are coordinates on the mid-surface of the shell, such that $z=$ constant and $\theta=$ constant are the lines of principal curvatures. Here $\theta$ will denote the circumferential coordinate and $z$-the longitudinal for cylindrically shaped shells. In the case of a straight circular cylinder $\theta$ and $z$ are the standard cylindrical coordinates. Let $\boldsymbol{r}(\theta, z)$ be the position vector of the shell's mid-surface. Introducing the normal coordinate $t$, we obtain the set of orthogonal curvilinear coordinates $(t, \theta, z)$, related to Cartesian coordinates via

$$
\boldsymbol{x}=\boldsymbol{R}(t, \theta, z)=\boldsymbol{r}(z, \theta)+\operatorname{tn}(z, \theta),
$$

where $\boldsymbol{n}$ is the outward unit normal, and $\boldsymbol{R}(t, \theta, z)$ is the position vector of a point in space with coordinates $(t, \theta, z)$. In this paper we will study shells of uniform thickness $h$, given in $(t, \theta, z)$ coordinates by

$$
\mathcal{C}_{h}=\left\{\boldsymbol{R}(t, \theta, z): z \in\left[L_{-}, L_{+}\right], t \in I_{h}, \theta \in[0, p)\right\}, \quad I_{h}=\left[-\frac{h}{2}, \frac{h}{2}\right] .
$$

We denote

$$
A_{z}^{2}=\left|\boldsymbol{r}_{, z}\right|^{2}, \quad A_{\theta}^{2}=\left|\boldsymbol{r}_{, \theta}\right|^{2}
$$

the two nonzero components $2^{2}$ of the metric tensor of the middle surface. The two principal curvatures will be denoted by $\kappa_{z}$ and $\kappa_{\theta}$. Their signs are chosen in such a way that $k_{z}$ and $k_{\theta}$ are positive for a barrel-shaped shells, like a sphere. The four functions $A_{\theta}, A_{z}, \kappa_{\theta}$, and $\kappa_{z}$ satisfy the Codazzi-Gauss relations (see e.g. [17, Section 1.1])

$$
\frac{\partial \kappa_{z}}{\partial \theta}=\left(\kappa_{\theta}-\kappa_{z}\right) \frac{A_{z, \theta}}{A_{z}}, \quad \frac{\partial \kappa_{\theta}}{\partial z}=\left(\kappa_{z}-\kappa_{\theta}\right) \frac{A_{\theta, z}}{A_{\theta}}
$$

\footnotetext{
${ }^{2}$ The principal directions are mutually orthogonal.
} 


$$
\frac{\partial}{\partial z}\left(\frac{A_{\theta, z}}{A_{z}}\right)+\frac{\partial}{\partial \theta}\left(\frac{A_{z, \theta}}{A_{\theta}}\right)=-A_{z} A_{\theta} \kappa_{z} \kappa_{\theta},
$$

and define the Levi-Civita connection on the middle surface of the shell via the following derivation formulas

$$
\begin{array}{lll}
\nabla_{\boldsymbol{e}_{z}} \boldsymbol{e}_{z}=-\frac{1}{A_{z} A_{\theta}} \frac{\partial A_{z}}{\partial \theta} \boldsymbol{e}_{\theta}-\kappa_{z} \boldsymbol{n}, & \nabla_{\boldsymbol{e}_{z}} \boldsymbol{e}_{\theta}=\frac{1}{A_{z} A_{\theta}} \frac{\partial A_{z}}{\partial \theta} \boldsymbol{e}_{z}, & \nabla_{\boldsymbol{e}_{z}} \boldsymbol{n}=\kappa_{z} \boldsymbol{e}_{z}, \\
\nabla_{\boldsymbol{e}_{\theta}} \boldsymbol{e}_{\theta}=-\frac{1}{A_{z} A_{\theta}} \frac{\partial A_{\theta}}{\partial z} \boldsymbol{e}_{z}-\kappa_{\theta} \boldsymbol{n}, & \nabla_{\boldsymbol{e}_{\theta} \boldsymbol{e}_{z}}=\frac{1}{A_{z} A_{\theta}} \frac{\partial A_{\theta}}{\partial z} \boldsymbol{e}_{\theta}, & \nabla_{\boldsymbol{e}_{\theta}} \boldsymbol{n}=\kappa_{\theta} \boldsymbol{e}_{\theta} .
\end{array}
$$

Using these formulas we can compute the components of $\operatorname{grad} \boldsymbol{u}$ in the orthonormal basis $\boldsymbol{e}_{t}$, $\boldsymbol{e}_{\theta}, \boldsymbol{e}_{z}$ :

$$
\operatorname{grad} \boldsymbol{u}=\left[\begin{array}{ccc}
u_{t, t} & \frac{u_{t, \theta}-A_{\theta} \kappa_{\theta} u_{\theta}}{A_{\theta}\left(1+t \kappa_{\theta}\right)} & \frac{u_{t, z}-A_{z} \kappa_{z} u_{z}}{A_{z}\left(1+t \kappa_{z}\right)} \\
u_{\theta, t} \frac{A_{z} u_{\theta, \theta}+A_{z} A_{\theta} \kappa_{\theta} u_{t}+A_{\theta, z} u_{z}}{A_{z} A_{\theta}\left(1+t \kappa_{\theta}\right)} & \frac{A_{\theta} u_{\theta, z}-A_{z, \theta} u_{z}}{A_{z} A_{\theta}\left(1+t \kappa_{z}\right)} \\
u_{z, t} & \frac{A_{z} u_{z, \theta}-A_{\theta, z} u_{\theta}}{A_{z} A_{\theta}\left(1+t \kappa_{\theta}\right)} & \frac{A_{\theta} u_{z, z}+A_{z} A_{\theta} \kappa_{z} u_{t}+A_{z, \theta} u_{\theta}}{A_{z} A_{\theta}\left(1+t \kappa_{z}\right)}
\end{array}\right] .
$$

We will now specialize to the particular case of zero Gaussian curvature $\kappa_{z}=0$. In this case, equations (2.2)-(2.3) can be solved explicitly in terms of four arbitrary smooth functions $B(z), a(\theta), b(\theta), c(\theta)$ :

$$
A_{z}=B^{\prime}(z), \quad A_{\theta}=a(\theta) B(z)+b(\theta), \quad \kappa_{\theta}=\frac{c(\theta)}{A_{\theta}} .
$$

We require that $A_{z}, A_{\theta}$ and $c(\theta)$ be strictly positive functions of their variables on the midsurface of the shell. Hence, for shells of zero Gaussian curvature the formula for grad $\boldsymbol{u}$ simplifies:

$$
\operatorname{grad} \boldsymbol{u}=\left[\begin{array}{ccc}
u_{t, t} & \frac{u_{t, \theta}-c(\theta) u_{\theta}}{A_{\theta}+t c(\theta)} & \frac{u_{t, z}}{A_{z}} \\
u_{\theta, t} & \frac{u_{\theta, \theta}+c(\theta) u_{t}+a(\theta) u_{z}}{A_{\theta}+t c(\theta)} & \frac{u_{\theta, z}}{A_{z}} \\
u_{z, t} & \frac{u_{z, \theta}-a(\theta) u_{\theta}}{A_{\theta}+t c(\theta)} & \frac{u_{z, z}}{A_{z}}
\end{array}\right]
$$

In the case of shells the thickness variable $t$ is uniformly small. We therefore introduce the simplified gradient

$$
\nabla \boldsymbol{u}=\left[\begin{array}{ccc}
u_{t, t} & \frac{u_{t, \theta}-c(\theta) u_{\theta}}{A_{\theta}} & \frac{u_{t, z}}{A_{z}} \\
u_{\theta, t} & \frac{u_{\theta, \theta}+c(\theta) u_{t}+a(\theta) u_{z}}{A_{\theta}} & \frac{u_{\theta, z}}{A_{z}} \\
u_{z, t} & \frac{u_{z, \theta}-a(\theta) u_{\theta}}{A_{\theta}} & \frac{u_{z, z}}{A_{z}}
\end{array}\right]
$$


We note in (2.6) the components $u_{t}, u_{\theta}$ and $u_{z}$ are still functions of $(t, \theta, z)$.

To be more specific we give two examples of zero Gaussian curvature shells: cylinders and cones. A cylinder is described by a simple, smooth closed curve of length $p$ in the $x y$-plane. Let $\boldsymbol{\rho}(\theta), \theta \in[0, p)$ be the position vector of this curve, parametrized by its arc-length. The position vector of the middle surface of the shell is then given by $\boldsymbol{r}(\theta, z)=\boldsymbol{\rho}(\theta)+z \boldsymbol{e}_{z}$, where $\boldsymbol{e}_{z}$ is the unit vector perpendicular to the $x y=$ plane, i.e. the unit vector in the $z$-direction. It is easy to verify that $\theta=$ constant and $z=$ constant are lines of curvature and $\kappa_{\theta}=\kappa(\theta)$ is the curvature of curve $\boldsymbol{\rho}(\theta)$ in the plane, whose sign is chosen to be positive for a circle.

A second example is a cone with vertex at the origin. A cone is described by a simple, smooth closed curve of length $p$ lying in the northern hemisphere of a unit sphere centered at the origin. Let $\boldsymbol{\sigma}(\theta), \theta \in[0, p)$ be the arc-length parametrization of this curve. In this case the middle surface of the shell is given by $\boldsymbol{r}(\theta, z)=z \boldsymbol{\sigma}(\theta)$. Once again, it is easy to verify that $\theta=$ constant and $z=$ constant are lines of curvature and

$$
\kappa_{\theta}=\frac{\left(\boldsymbol{\sigma}(\theta), \boldsymbol{\sigma}^{\prime}(\theta), \boldsymbol{\sigma}^{\prime \prime}(\theta)\right)}{z}
$$

where $(\boldsymbol{a}, \boldsymbol{b}, \boldsymbol{c})=\boldsymbol{a} \cdot(\boldsymbol{b} \times \boldsymbol{c})$ is the triple-product of 3 vectors in space. We summarize the data for cylinders and cones in Table 1 .

\begin{tabular}{|c|c|c|c|c|}
\hline & $B(z)$ & $a(\theta)$ & $b(\theta)$ & $c(\theta)$ \\
\hline cylinders & $z$ & 0 & 1 & $\kappa(\theta)$ \\
\hline cones & $z$ & 1 & 0 & $\left(\boldsymbol{\sigma}(\theta), \boldsymbol{\sigma}^{\prime}(\theta), \boldsymbol{\sigma}^{\prime \prime}(\theta)\right)$ \\
\hline
\end{tabular}

Table 1: Functions $B(z), a(\theta), b(\theta)$ and $c(\theta)$ for cylinders and cones.

In this paper all norms $\|\cdot\|$ are $L^{2}$ norms. However because of the curvilinear coordinates we will use several different flavors of the $L^{2}$ inner product and the corresponding norm. For $f, g: \mathcal{C}_{h} \rightarrow \mathbb{R}$ we define the $L^{2}$ inner product

$$
(f, g)_{\mathcal{C}_{h}}=\int_{\mathcal{C}_{h}} f(\boldsymbol{x}) g(\boldsymbol{x}) d \boldsymbol{x}=\int_{I_{h}} \int_{l}^{L} \int_{0}^{p} A_{z} A_{\theta} f g d \theta d z d t
$$

which gives rise to the norm $\|f\|$.

$$
\|f\|^{2}=(f, f)=\int_{I_{h}} \int_{l}^{L} \int_{0}^{p} A_{z} A_{\theta} f^{2} d \theta d z d t .
$$

In cross-sections $\theta=$ constant we use

$$
\|f\|_{\theta}^{2}=\int_{I_{h}} \int_{l}^{L} A_{z} f(t, \theta, z)^{2} d z d t .
$$

We will also use the Euclidean version of the norm on cross-sections

$$
\|f\|_{0}^{2}=\int_{\alpha_{1}}^{\alpha_{2}} \int_{\beta_{1}}^{\beta_{2}} f(\alpha, \beta, \gamma) d \alpha d \beta
$$


where $\{\alpha, \beta, \gamma\}=\{t, \theta, z\}$ and $\alpha_{i}, \beta_{i}, i=1,2$ are the corresponding limits of integration. In each case it will be clear which variable $t, \theta$ or $z$ plays the role of the fixed variable $\gamma$. Of course, due to uniform positivity of $A_{\theta}$ and $A_{z}$ the norms $\|f\|_{\gamma}$ and $\|f\|_{0}$ are obviously equivalent. In particular, all inequalities involving one type of norm will also be valid for another. Finally, all constants that are independent of $\boldsymbol{u}$ and $h$ will be denoted by $C$. Once this is understood, such abuse of notation does not lead to any ambiguity.

\section{Main results}

We formulate our Korn inequalities for vector fields $\boldsymbol{u}$ satisfying specific boundary conditions at the two edges of the shell. We define

$$
\begin{aligned}
& V_{h}^{1}=\left\{\boldsymbol{\phi} \in W^{1,2}\left(\mathcal{C}_{h} ; \mathbb{R}^{3}\right): \boldsymbol{\phi}(r, \theta, 0)=\mathbf{0}, \phi_{r}(r, \theta, L)=\phi_{\theta}(r, \theta, L)=0\right\} \\
& \quad \cap\left\{\boldsymbol{\phi} \in W^{1,2}\left(\mathcal{C}_{h} ; \mathbb{R}^{3}\right): \boldsymbol{\phi}_{\theta}, \boldsymbol{\phi}_{z} \text { are } p \text { - periodic in } \theta\right\} .
\end{aligned}
$$

and

$$
\begin{aligned}
V_{h}^{2}=\left\{\boldsymbol{\phi} \in W^{1,2}\left(\mathcal{C}_{h} ; \mathbb{R}^{3}\right): \phi_{\theta}(r, \theta, 0)=\phi_{z}(r, \theta, 0)=\phi_{\theta}(r, \theta, L)=\phi_{z}(r, \theta, L)=0\right\} \\
\cap\left\{\boldsymbol{\phi} \in W^{1,2}\left(\mathcal{C}_{h} ; \mathbb{R}^{3}\right): \boldsymbol{\phi}_{\theta}, \boldsymbol{\phi}_{z} \text { are } p-\text { periodic in } \theta\right\} .
\end{aligned}
$$

We state our main results as a sequence of related theorems.

Theorem 3.1. Suppose $\kappa_{z}=0$ on $\mathcal{C}_{h}$. Then there exist a constant $C$, independent of $h$, such that for every $\boldsymbol{u} \in V_{h}^{1}$

$$
\begin{aligned}
\|\operatorname{grad} \boldsymbol{u}\|^{2} \leq & C\left(\frac{\left\|u_{t}\right\|\left\|(\operatorname{grad} \boldsymbol{u})_{\mathrm{sym}}\right\|}{h}+\left\|(\operatorname{grad} \boldsymbol{u})_{\mathrm{sym}}\right\|^{2}\right) \\
& \|\operatorname{grad} \boldsymbol{u}\|^{2} \leq \frac{C}{h^{2}}\left\|(\operatorname{grad} \boldsymbol{u})_{\mathrm{sym}}\right\|^{2}
\end{aligned}
$$

for all $h \in(0,1)$.

If additionally, the curvature $\kappa_{\theta}$ vanishes on an open subset of the middle surface of the shell then, according to Theorem 3.3 the bound in (3.4) is asymptotically sharp as $h \rightarrow 0$. If the curvature $\kappa_{\theta}$ does not change sign (i.e. stays uniformly positive for cylindrically-shaped shells), then the first Korn inequality (3.4) can be improved.

Theorem 3.2. Suppose that $\kappa_{z}=0$ and $\kappa_{\theta}>0$ on $\mathcal{C}_{h}$. Then there exist a constant $C$, independent of $h$, such that for every $\boldsymbol{u} \in V_{h}^{1} \cup V_{h}^{2}$ inequalities (3.3) and

$$
\|\operatorname{grad} \boldsymbol{u}\|^{2} \leq \frac{C}{h \sqrt{h}}\left\|(\operatorname{grad} \boldsymbol{u})_{\mathrm{sym}}\right\|^{2}
$$

hold for all $h \in(0,1)$.

In fact, inequalities (3.3) and (3.5) are asymptotically sharp. 
THEOREM 3.3 (Existence of optimal ansatz).

(i) Suppose that assumptions of Theorem 3.1 are satisfied. Suppose, additionally that the curvature $\kappa_{\theta}$ vanishes on an open subset of the middle surface of the shell. Then there exist functions $\boldsymbol{u}^{h} \in V_{h}^{1} \cap V_{h}^{2}$ and a constant $C$, independent of $h$, for which

$$
\left\|\left(\operatorname{grad} \boldsymbol{u}^{h}\right)_{\text {sym }}\right\|^{2} \leq C h^{2}\left\|\operatorname{grad} \boldsymbol{u}^{h}\right\|^{2} .
$$

(ii) Suppose that assumptions of Theorem 3.2 are satisfied. Then there exist functions $\boldsymbol{u}^{h} \in V_{h}^{1} \cap V_{h}^{2}$ and a constant $C$, independent of $h$, for which

$$
\left\|\left(\operatorname{grad} \boldsymbol{u}^{h}\right)_{\mathrm{sym}}\right\|^{2} \leq C h \sqrt{h}\left\|\operatorname{grad} \boldsymbol{u}^{h}\right\|^{2} .
$$

Remark 3.4. Our results are formulated for shells cut along the coordinate surfaces. However, they are also valid for any shell $\mathcal{C}_{h}$, bounded by the surfaces $z=Z_{ \pm}(t, \theta)$, where the spaces $V_{h}^{1}, V_{h}^{2}$ are defined by (3.1), (3.2), respectively, except the indicated components of $\boldsymbol{u}$ vanish on the surfaces $z=Z_{ \pm}(t, \theta)$, instead of $z=L_{ \pm}$. This is because there exists shells $\mathcal{C}_{h}^{-} \subset \mathcal{C}_{h} \subset \mathcal{C}_{h}^{+}$, such that the shells $\mathcal{C}_{h}^{ \pm}$are bounded by surfaces $z=$ constant. But then the ansatz from Theorem 3.3 supported in $\mathcal{C}_{h}^{-}$gives an upper bound on the Korn constant of $\mathcal{C}_{h}$ that scales as $h^{3 / 2}$ (or as $h^{2}$ ). At the same time every function in $V_{h}^{1}$ or $V_{h}^{2}$ of $\mathcal{C}_{h}$ can be extended (by extending the relevant components of $\boldsymbol{u}$ by zero) to a function in $V_{h}^{1}$ or $V_{h}^{2}$ of $\mathcal{C}_{h}^{+}$, giving the lower bound on the Korn constant that scales as $h^{3 / 2}$ (or as $h^{2}$ ).

Remark 3.5. Note, that the periodicity condition in the $\theta$-direction in (3.1)-(3.2) will be automatically satisfied for cylindrically-shaped shells. However, it is easy to check, that our main results hold (the proofs below go through) for different class or displacements $\boldsymbol{u}$ satisfying Robin boundary conditions on the thin faces of the shell. For instance, one can assume the condition

$$
\left\{\boldsymbol{\phi} \in W^{1,2}\left(\mathcal{C}_{h} ; \mathbb{R}^{3}\right): \phi_{\theta}(t, 0, z)=\phi_{\theta}(t, p, z)=0\right\} .
$$

on the thin edges of the shell in the $\theta$ direction instead of the p-perioditicy of the $\theta$ and the $z$ components in (3.1) and (3.2). Note, also, that the boundary condition (3.8) is of Dirichlet type, but it can be rewritten as a Neumann condition $\boldsymbol{\Phi} \cdot n=0$ on the thin edges of the shell in the $\theta$ direction.

\section{Proofs of Theorems 3.1 and 3.2}

Our the strategy is to prove first-and-a-half Korn inequality for the simplified version $\nabla \boldsymbol{u}$ of grad $\boldsymbol{u}$, given by (2.6)

$$
\|\nabla \boldsymbol{u}\|^{2} \leq C\left(\frac{\left\|u_{t}\right\|\|e(\boldsymbol{u})\|}{h}+\|e(\boldsymbol{u})\|^{2}\right)
$$

where

$$
e(\boldsymbol{u})=\frac{1}{2}\left(\nabla \boldsymbol{u}+(\nabla \boldsymbol{u})^{T}\right)
$$


We then show that (4.1) implies (3.3). In order to prove (4.1) we apply the method, introduced in [3], of assembling (4.1) from the analogous two-dimensional inequalities corresponding to the three coordinate surface cross-sections of the shell. Most of the proof is done under the common assumptions of Theorems 3.1 and 3.2. In other words, we assume that $\boldsymbol{u} \in V_{h}^{1} \cup V_{h}^{2}$ and we do not make any assumptions on the sign of $\kappa_{\theta}$, until we say otherwise.

\subsection{The $t=$ const cross-section}

The Korn-type inequality corresponding to $t=$ const cross-section involves $\theta \theta, \theta z, z \theta$, and $z z$ components of the gradient. The first-and-a-half Korn inequality in this case is stated in the following lemma.

LEMMA 4.1.

$$
\left\|(\nabla \boldsymbol{u})_{\theta z}\right\|^{2}+\left\|(\nabla \boldsymbol{u})_{z \theta}\right\|^{2} \leq C\|e(\boldsymbol{u})\|\left(\|e(\boldsymbol{u})\|+\left\|u_{t}\right\|\right) .
$$

Proof. Observing that

$$
\left\|(\nabla \boldsymbol{u})_{\theta z}\right\|^{2}+\left\|(\nabla \boldsymbol{u})_{z \theta}\right\|^{2}=4\left\|e(\boldsymbol{u})_{\theta z}\right\|^{2}-2\left((\nabla \boldsymbol{u})_{\theta z},(\nabla \boldsymbol{u})_{z \theta}\right)_{\mathcal{C}_{h}},
$$

we conclude that it is sufficient to prove

$$
\left|\left((\nabla \boldsymbol{u})_{\theta z},(\nabla \boldsymbol{u})_{z \theta}\right)_{\mathcal{C}_{h}}\right| \leq C\|e(\boldsymbol{u})\|\left(\|e(\boldsymbol{u})\|+\left\|u_{t}\right\|\right)
$$

We have, that

$$
\left((\nabla \boldsymbol{u})_{\theta z},(\nabla \boldsymbol{u})_{z \theta}\right)_{\mathcal{C}_{h}}=\int_{I_{h}} \int_{l}^{L} \int_{0}^{p} A_{\theta} A_{z} \frac{u_{\theta, z}}{A_{z}} \frac{u_{z, \theta}-a(\theta) u_{\theta}}{A_{\theta}} d \theta d z d t=\int_{I_{h}}\left(I_{1}(t)-I_{2}(t)\right) d t
$$

where

$$
I_{1}(t)=\int_{l}^{L} \int_{0}^{p} u_{\theta, z} u_{z, \theta} d \theta d z
$$

and

$$
I_{2}(t)=\int_{l}^{L} \int_{0}^{p} a(\theta) u_{\theta} u_{\theta, z} d \theta d z=\frac{1}{2} \int_{0}^{p} a(\theta) \int_{l}^{L}\left(u_{\theta}^{2}\right)_{, z} d z=0,
$$

since $u_{\theta}=0$ at $z=l$ and $z=L$ in both $V_{h}^{1}$ and $V_{h}^{2}$.

Let us estimate $I_{1}(t)$. The idea is to observe that in both $V_{h}^{1}$ and $V_{h}^{2}$,

$$
\int_{l}^{L} \int_{0}^{p} u_{\theta, z} u_{z, \theta} d \theta d z=-\int_{l}^{L} \int_{0}^{p} u_{\theta} u_{z, \theta z} d \theta d z=\int_{l}^{L} \int_{0}^{p} u_{\theta, \theta} u_{z, z} d \theta d z
$$

and then express $u_{\theta, \theta}$ and $u_{z, z}$ in terms of $(\nabla \boldsymbol{u})_{\theta \theta}=e(\boldsymbol{u})_{\theta \theta}$ and $(\nabla \boldsymbol{u})_{z z}=e(\boldsymbol{u})_{z z}$, respectively. Thus,

$$
\int_{I_{h}} I_{1}(t) d t=\left(e(\boldsymbol{u})_{z z}, e(\boldsymbol{u})_{\theta \theta}-\frac{a(\theta)}{A_{\theta}} u_{z}-\kappa_{\theta} u_{t}\right) .
$$


Applying the Schwartz inequality we obtain

$$
\left|\int_{I_{h}} I_{1}(t) d t\right| \leq C\|e(\boldsymbol{u})\|\left(\|e(\boldsymbol{u})\|+\left\|u_{t}\right\|+\left\|u_{z}\right\|\right) .
$$

By the Poincaré inequality

$$
\left\|u_{z}\right\| \leq C\left\|u_{z, z}\right\| \leq C\|e(\boldsymbol{u})\| .
$$

Using this inequality in (4.5) we obtain the desired bound (4.2).

\subsection{The $\theta=$ const cross-section}

LEMMA 4.2.

$$
\left\|(\nabla \boldsymbol{u})_{t z}\right\|^{2}+\left\|(\nabla \boldsymbol{u})_{z t}\right\|^{2} \leq C\left(\frac{\left\|u_{t}\right\| \cdot\|e(\boldsymbol{u})\|}{h}+\|e(\boldsymbol{u})\|^{2}\right) .
$$

Let us show that Lemma 4.2 is an immediate consequence of the same two-dimensional inequality in Cartesian coordinates, proved in [3. Theorem 3.1]. It states that if $h \in(0,1)$, $\phi=(u, v) \in H^{1}\left(I_{h} \times\left[L_{-}, L_{+}\right] ; \mathbb{R}^{2}\right)$ satisfies $v(x, l)=0, x \in I_{h}$ in the sense of traces, then

$$
\left\|\nabla^{c} \boldsymbol{\phi}\right\|_{0}^{2} \leq C\left\|e^{c}(\boldsymbol{\phi})\right\|_{0}\left(\frac{\|u\|_{0}}{h}+\left\|e^{c}(\boldsymbol{\phi})\right\|_{0}\right)
$$

where

$$
\nabla^{c} \boldsymbol{\phi}=\left[\begin{array}{ll}
u_{, x} & u_{, y} \\
v_{, x} & v_{, y}
\end{array}\right], \quad e^{c}(\boldsymbol{\phi})=\frac{1}{2}\left(\nabla^{c} \boldsymbol{\phi}+\left(\nabla^{c} \boldsymbol{\phi}\right)^{T}\right) .
$$

Lemma 4.2 says that the same statement holds in our curvilinear coordinates, where $\nabla \boldsymbol{u}$ is given by (2.6).

Proof of Lemma 4.2. We first prove inequality (4.7) for each fixed $\theta \in[0, p]$ :

$$
\left\|(\nabla \boldsymbol{u})_{t z}\right\|_{\theta}^{2}+\left\|(\nabla \boldsymbol{u})_{z t}\right\|_{\theta}^{2} \leq C\left(\frac{\left\|u_{t}\right\|_{\theta} \cdot\left\|e_{\theta}(\boldsymbol{u})\right\|_{\theta}}{h}+\left\|e_{\theta}(\boldsymbol{u})\right\|_{\theta}^{2}\right)
$$

where

$$
\|f\|_{\theta}^{2}=\int_{I_{h}} \int_{l}^{L} A_{z} f(t, \theta, z)^{2} d z d t, \quad e_{\theta}(\boldsymbol{u})=\left[\begin{array}{cc}
u_{t, t} & \frac{1}{2}\left(\frac{u_{t, z}}{A_{z}}+u_{z, t}\right) \\
\frac{1}{2}\left(\frac{u_{t, z}}{A_{z}}+u_{z, t}\right) & \frac{u_{z, z}}{A_{z}}
\end{array}\right] .
$$

Then inequality (4.7) is obtained from (4.9) by the Cauchy-Schwartz inequality

$$
\int_{0}^{p} A_{\theta}\|f\|_{\theta}\|g\|_{\theta} d \theta \leq\|f\|\|g\|,
$$


which becomes equality if $f=g$. Let

$$
\boldsymbol{G}=\left[\begin{array}{cc}
u_{t, t} & u_{t, z} \\
A_{z} u_{z, t} & \left(A_{z} u_{z}\right)_{, z}
\end{array}\right], \quad \boldsymbol{E}=\frac{1}{2}\left(\boldsymbol{G}+\boldsymbol{G}^{T}\right) .
$$

Then, by (4.8), applied to $\phi=\left(u_{t}, A_{z} u_{z}\right)$, we obtain

$$
\|\boldsymbol{G}\|_{0}^{2} \leq C\|\boldsymbol{E}\|_{0}\left(\frac{\left\|u_{t}\right\|_{0}}{h}+\|\boldsymbol{E}\|_{0}\right) .
$$

By uniform positivity and boundedness of $A_{\theta}, A_{z}$ norms $\|\cdot\|_{0}$ and $\|\cdot\|_{\theta}$ are equivalent. Hence,

$$
\left\|(\nabla \boldsymbol{u})_{t z}\right\|_{\theta}^{2}+\left\|(\nabla \boldsymbol{u})_{z t}\right\|_{\theta}^{2} \leq C\left(\left\|\boldsymbol{G}_{t z}\right\|_{0}^{2}+\left(\left\|\boldsymbol{G}_{z t}\right\|_{0}^{2}\right) \leq C\|\boldsymbol{G}\|_{0}^{2}\right.
$$

Applying (4.10), we prove the lemma, if we show that

$$
\|\boldsymbol{E}\|_{0} \leq C\left\|e_{\theta}(\boldsymbol{u})\right\|_{\theta}
$$

We estimate

$$
\|\boldsymbol{E}\|_{0}^{2} \leq C\left(\left\|u_{t, t}\right\|_{\theta}^{2}+\left\|u_{z, z}\right\|_{\theta}^{2}+\left\|u_{z}\right\|_{\theta}^{2}\right)+\frac{1}{2}\left\|u_{t, z}+A_{z} u_{z, t}\right\|_{0}^{2} .
$$

By the Poincaré inequality $\left\|u_{z}\right\|_{\theta}^{2} \leq\left\|u_{z, z}\right\|_{\theta}^{2}$, so that

$$
\|\boldsymbol{E}\|_{0}^{2} \leq C\left\|e_{\theta}(\boldsymbol{u})\right\|_{\theta}^{2}+\frac{1}{2}\left\|u_{t, z}+A_{z} u_{z, t}\right\|_{0}^{2} .
$$

It remains to observe that

$$
\left\|u_{t, z}+A_{z} u_{z, t}\right\|_{0}=\left\|\sqrt{A_{z}}\left(\frac{u_{t, z}}{A_{z}}+u_{z, t}\right)\right\|_{\theta} \leq C\left\|e_{\theta}(\boldsymbol{u})\right\|_{\theta} .
$$

\subsection{The $z=$ const cross-section}

\section{LEMMA 4.3.}

$$
\left\|(\nabla \boldsymbol{u})_{t \theta}\right\|^{2}+\left\|(\nabla \boldsymbol{u})_{\theta t}\right\|^{2} \leq C\left(\frac{\left\|u_{t}\right\| \cdot\|e(\boldsymbol{u})\|}{h}+\|e(\boldsymbol{u})\|^{2}\right) .
$$

Proof. As before, we will show that that (4.12) is a consequence of a two-dimensional Korntype inequality. However, before we can proceed with this strategy, we observe that the term with $u_{z}$ in the $\theta \theta$-component of $\nabla \boldsymbol{u}$ can be easily discarded due to the Poincaré inequality (4.6). Indeed, suppose we have proved (4.12), where $e(\boldsymbol{u})$ is replaced with

$$
e^{\prime}(\boldsymbol{u})=\left[\begin{array}{cc}
e(\boldsymbol{u})_{t t} & e(\boldsymbol{u})_{t \theta} \\
e(\boldsymbol{u})_{t \theta} & e^{\prime}(\boldsymbol{u})_{\theta \theta}
\end{array}\right]
$$


where

$$
e^{\prime}(\boldsymbol{u})_{\theta \theta}=\frac{u_{\theta, \theta}+c(\theta) u_{t}}{A_{\theta}}
$$

Then

$$
\left\|e^{\prime}(\boldsymbol{u})\right\| \leq\|e(\boldsymbol{u})\|+C\left\|u_{z}\right\| \leq C\|e(\boldsymbol{u})\|
$$

so that

$$
\left\|(\nabla \boldsymbol{u})_{t \theta}\right\|^{2}+\left\|(\nabla \boldsymbol{u})_{\theta t}\right\|^{2} \leq C\left(\frac{\left\|u_{t}\right\| \cdot\left\|e^{\prime}(\boldsymbol{u})\right\|}{h}+\left\|e^{\prime}(\boldsymbol{u})\right\|^{2}\right) \leq C\left(\frac{\left\|u_{t}\right\| \cdot\|e(\boldsymbol{u})\|}{h}+\|e(\boldsymbol{u})\|^{2}\right) .
$$

Next we prove the two-dimensional Korn-type inequality

LEMMA 4.4. Let

$$
V=\left\{\phi=(u, v) \in H_{\mathrm{loc}}^{1}\left(\left[-\frac{h}{2}, \frac{h}{2}\right] \times \mathbb{R}\right): u(x, y) \text { is } p-\text { periodic }\right\}
$$

Let

$$
\boldsymbol{G}=\left[\begin{array}{ll}
u_{, x} & \alpha(y) u_{, y}-\kappa(y) v \\
v_{, x} & \alpha(y) v_{, y}+\kappa(y) u
\end{array}\right], \quad \boldsymbol{E}=\frac{1}{2}\left(\boldsymbol{G}+\boldsymbol{G}^{T}\right)
$$

Then,

$$
\|\boldsymbol{G}\|_{0}^{2} \leq C\left(\frac{\|u\|_{0}\|\boldsymbol{E}\|_{0}}{h}+\|\boldsymbol{E}\|_{0}^{2}+\|\phi\|_{0}^{2}\right) .
$$

This lemma is proved in Section 5. If we apply this lemma to $\phi(t, \theta)=\left(u_{t}(t, \theta, z), u_{\theta}(t, \theta, z)\right)$ (for each fixed value of $z$ ) and then integrate over $z \in\left[L_{-}, L_{+}\right]$, we obtain the inequality (taking into account the equivalence between the curvilinear norm (2.7) and the Euclidean norm)

$$
\left\|(\nabla \boldsymbol{u})_{t \theta}\right\|^{2}+\left\|(\nabla \boldsymbol{u})_{\theta t}\right\|^{2} \leq C\left(\frac{\left\|u_{t}\right\| \cdot\|e(\boldsymbol{u})\|}{h}+\|e(\boldsymbol{u})\|^{2}+\left\|u_{t}\right\|^{2}+\left\|u_{\theta}\right\|^{2}\right) .
$$

By the Poincaré inequality and (4.2) we obtain

$$
\left\|u_{\theta}\right\|^{2} \leq C\left\|(\nabla \boldsymbol{u})_{\theta z}\right\|^{2} \leq C\|e(\boldsymbol{u})\|\left(\|e(\boldsymbol{u})\|+\left\|u_{t}\right\|\right) .
$$

At this point the different assumptions in Theorems 3.1 and 3.2 become important.

If $\boldsymbol{u} \in V_{h}^{1}$ then we can just use the Poincaré inequality and (4.7)

$$
\left\|u_{t}\right\|^{2} \leq C\left\|(\nabla \boldsymbol{u})_{t z}\right\|^{2} \leq C\left(\frac{\left\|u_{t}\right\| \cdot\|e(\boldsymbol{u})\|}{h}+\|e(\boldsymbol{u})\|^{2}\right) .
$$

Under the assumptions of Theorem 3.2 the Poincaré inequality cannot be used. Instead we estimate expressing $u_{t}$ in terms of $e^{\prime}(\boldsymbol{u})_{\theta \theta}$ :

$$
u_{t}=\frac{A_{\theta} e^{\prime}(\boldsymbol{u})_{\theta \theta}-u_{\theta, \theta}}{A_{\theta} \kappa_{\theta}} .
$$


Multiplying both sides of (4.17) by $u_{t}$ and integrating by parts (without writing integrals), we obtain

$$
u_{t}^{2}=\frac{u_{t} e^{\prime}(\boldsymbol{u})_{\theta \theta}}{\kappa_{\theta}}-\frac{\partial}{\partial \theta}\left(\frac{u_{\theta} u_{t}}{A_{\theta} \kappa_{\theta}}\right)+u_{\theta} u_{t} \frac{\partial}{\partial \theta}\left(\frac{1}{A_{\theta} \kappa_{\theta}}\right)+\frac{u_{\theta} u_{t, \theta}}{A_{\theta} \kappa_{\theta}} .
$$

Finally, replacing $u_{t, \theta}$ in the formula above by

$$
u_{t, \theta}=A_{\theta}(\nabla \boldsymbol{u})_{t \theta}+c(\theta) u_{\theta}
$$

and using periodicity in $\theta$ we obtain the estimate, taking into account the equivalence of $\|\cdot\|_{0}$ and $\|\cdot\|$ norms

$$
\left\|u_{t}\right\|^{2} \leq C\left(\left\|u_{t}\right\|\|e(\boldsymbol{u})\|+\left\|u_{t}\right\|\left\|u_{\theta}\right\|+\left\|u_{\theta}\right\|^{2}+\left\|(\nabla \boldsymbol{u})_{t \theta}\right\|\left\|u_{\theta}\right\|\right) .
$$

Hence, using the inequality $C a b \leq a^{2} / 2+C^{2} b^{2} / 2$ (several times) and replacing $\left\|u_{\theta}\right\|^{2}$ by its estimate from (4.15), we obtain the bound.

$$
\left\|u_{t}\right\|^{2} \leq C\left(\|e(\boldsymbol{u})\|^{2}+\left\|(\nabla \boldsymbol{u})_{t \theta}\right\|\left\|u_{\theta}\right\|\right) .
$$

Using this inequality to estimate the term $\left\|u_{t}\right\|^{2}$ in (4.14) we obtain the desired inequality (4.12).

\subsection{Conclusion of the proof}

Combining the estimates (4.2), (4.7) and (4.12) we arrive at (4.1). However, $\nabla \boldsymbol{u}$ and $e(\boldsymbol{u})$ are the simplified versions of $\operatorname{grad} \boldsymbol{u}$ and $(\operatorname{grad} \boldsymbol{u})_{\text {sym. }}$. Thus, we need to show that (3.3) follows from (4.1). Under the assumptions of Theorems 3.1 it is a consequence of (4.16), while under the assumptions of Theorems 3.2 it is a consequence of (4.18).

The main observation in either case is that components of $\operatorname{grad} \boldsymbol{u}$ and $\nabla \boldsymbol{u}$ are multiples of one another with coefficients that are independent of $\boldsymbol{u}$. Thus, by direct calculation, we estimate

$$
\|\operatorname{grad} \boldsymbol{u}-\nabla \boldsymbol{u}\| \leq C h\|\operatorname{grad} \boldsymbol{u}\|, \quad \text { for all } \boldsymbol{u} \in H^{1}\left(\mathcal{C}_{h}\right),
$$

from which we get additionally,

$$
\left\|(\operatorname{grad} \boldsymbol{u})_{\mathrm{sym}}-e(\boldsymbol{u})\right\| \leq\|\operatorname{grad} \boldsymbol{u}-\nabla \boldsymbol{u}\| \leq C h\|\operatorname{grad} \boldsymbol{u}\|, \quad \text { for all } \quad \boldsymbol{u} \in H^{1}\left(\mathcal{C}_{h}\right) .
$$

Proof of Theorem 3.1. Observe, that $t$, $t z, z t$ and $z z$ components of $\operatorname{grad} \boldsymbol{u}$ and $\nabla \boldsymbol{u}$ coincide. The analysis for the cross section $\theta=$ const involved only these components of the gradient. Thus, estimate (4.7) holds for $\operatorname{grad} \boldsymbol{u}$ in place of $\nabla \boldsymbol{u}$. This implies (4.16) for $(\operatorname{grad} \boldsymbol{u})_{\mathrm{sym}}$ in place of $e(\boldsymbol{u})$, i.e., we have

$$
\left\|u_{t}\right\| \leq \frac{\left\|(\operatorname{grad} \boldsymbol{u})_{\mathrm{sym}}\right\|}{h} .
$$

This allows us to show that (4.1) implies (3.3). Combining (4.1), (4.19) and (4.20), we obtain

$$
\|\operatorname{grad} \boldsymbol{u}\|^{2} \leq C\left(\frac{\left\|u_{t}\right\|\left\|(\operatorname{grad} \boldsymbol{u})_{\mathrm{sym}}\right\|}{h}+\left\|u_{t}\right\|\|\operatorname{grad} \boldsymbol{u}\|+\left\|(\operatorname{grad} \boldsymbol{u})_{\mathrm{sym}}\right\|^{2}\right) .
$$


Estimating

$$
C\left\|u_{t}\right\|\|\operatorname{grad} \boldsymbol{u}\| \leq \frac{1}{2}\|\operatorname{grad} \boldsymbol{u}\|^{2}+\frac{C^{2}}{2}\left\|u_{t}\right\|^{2}
$$

we get

$$
\|\operatorname{grad} \boldsymbol{u}\|^{2} \leq C\left(\frac{\left\|u_{t}\right\|\left\|(\operatorname{grad} \boldsymbol{u})_{\text {sym }}\right\|}{h}+\left\|u_{t}\right\|^{2}+\left\|(\operatorname{grad} \boldsymbol{u})_{\text {sym }}\right\|^{2}\right) .
$$

Finally, by (4.21)

$$
\left\|u_{t}\right\|^{2} \leq \frac{\left\|u_{t}\right\|\left\|(\operatorname{grad} \boldsymbol{u})_{\mathrm{sym}}\right\|}{h}
$$

and (3.3) follows. Combining estimates (4.21) and (3.3) we obtain (3.4).

Proof of Theorem 3.2. In this case we proceed in the same way as in [3], proving the following lemma.

LEMMA 4.5. Inequalities 4.1), 4.15), and 4.18) taken together, imply

$$
\|\nabla \boldsymbol{u}\|^{2} \leq \frac{C}{h \sqrt{h}}\|e(\boldsymbol{u})\|^{2}
$$

We postpone the proof of this virtually algebraic lemma to finish the proof of (3.3).

Combining (4.23) with (4.20) we obtain

$$
\|\operatorname{grad} \boldsymbol{u}\|^{2} \leq C\|\nabla \boldsymbol{u}\|^{2} \leq \frac{C}{h \sqrt{h}}\left(\left\|(\operatorname{grad} \boldsymbol{u})_{\text {sym }}\right\|^{2}+h^{2}\|\operatorname{grad} \boldsymbol{u}\|^{2}\right),
$$

proving the first Korn inequality (3.5). Now, inequality (4.1) and (3.5) imply (3.3). Indeed, Using the estimate

$$
\left\|u_{t}\right\|\|\operatorname{grad} \boldsymbol{u}\| \leq \frac{C\left\|u_{t}\right\|\left\|(\operatorname{grad} \boldsymbol{u})_{\mathrm{sym}}\right\|}{h^{3 / 4}} \leq \frac{C\left\|u_{t}\right\|\left\|(\operatorname{grad} \boldsymbol{u})_{\mathrm{sym}}\right\|}{h}
$$

in (4.22) we obtain (3.3).

Proof of Lemma 4.5. We begin with the inequality (4.18),

$$
\left\|u_{t}\right\|^{2} \leq C\left(\|e(\boldsymbol{u})\|^{2}+2\|\nabla \boldsymbol{u}\|\left\|u_{\theta}\right\|\right) \leq C\left(\|e(\boldsymbol{u})\|^{2}+\epsilon^{2}\|\nabla \boldsymbol{u}\|^{2}+\frac{1}{\epsilon^{2}}\left\|u_{\theta}\right\|^{2}\right)
$$

for any $\epsilon>0$. The small parameter $\epsilon \in(0,1)$ will be chosen later to optimize the resulting inequality. Estimating $\left\|u_{\theta}\right\|^{2}$ by (4.15) we obtain for sufficiently small $\epsilon$,

$$
\left\|u_{t}\right\|^{2} \leq C\left(\|e(\boldsymbol{u})\|^{2}+\epsilon^{2}\|\nabla \boldsymbol{u}\|^{2}+\frac{1}{\epsilon^{2}}\left(\|e(\boldsymbol{u})\|^{2}+\|e(\boldsymbol{u})\|\left\|u_{t}\right\|\right)\right)
$$

Estimating

$$
\frac{C}{\epsilon^{2}}\|e(\boldsymbol{u})\|\left\|u_{t}\right\| \leq \frac{1}{2}\left\|u_{t}\right\|^{2}+\frac{C^{2}}{2 \epsilon^{4}}\|e(\boldsymbol{u})\|^{2},
$$


we obtain

$$
\left\|u_{t}\right\|^{2} \leq C\left(\frac{\|e(\boldsymbol{u})\|^{2}}{\epsilon^{4}}+\epsilon^{2}\|\nabla \boldsymbol{u}\|^{2}\right)
$$

Thus,

$$
\left\|u_{t}\right\| \leq C\left(\frac{\|e(\boldsymbol{u})\|}{\epsilon^{2}}+\epsilon\|\nabla \boldsymbol{u}\|\right)
$$

Substituting this inequality into (4.1), we obtain

$$
\|\nabla \boldsymbol{u}\|^{2} \leq C\left(\frac{\|e(\boldsymbol{u})\|^{2}}{h \epsilon^{2}}+\frac{\epsilon\|\nabla \boldsymbol{u}\|\|e(\boldsymbol{u})\|}{h}\right) .
$$

Estimating

$$
\frac{C \epsilon\|\nabla \boldsymbol{u}\|\|e(\boldsymbol{u})\|}{h} \leq \frac{1}{2}\|\nabla \boldsymbol{u}\|^{2}+\frac{C^{2} \epsilon^{2}\|e(\boldsymbol{u})\|^{2}}{h^{2}},
$$

we obtain the inequality

$$
\|\nabla \boldsymbol{u}\|^{2} \leq C\left(\frac{1}{h \epsilon^{2}}+\frac{\epsilon^{2}}{h^{2}}\right)\|e(\boldsymbol{u})\|^{2} .
$$

We now choose $\epsilon=h^{1 / 4}$ to minimize the upper bound and obtain (4.23).

\section{The two-dimensional Korn-type inequality}

In this section we prove the following two-dimensional Korn-type inequality.

THEOREM 5.1. Let

$$
V=\left\{\boldsymbol{\phi}=(u, v) \in H_{\mathrm{loc}}^{1}\left(\left[-\frac{h}{2}, \frac{h}{2}\right] \times[0, p]\right): u(x, y) \text { is } p \text {-periodic }\right\}
$$

and let

$$
\boldsymbol{G}(\boldsymbol{\phi})=\left[\begin{array}{cc}
u_{, x} & a_{1}(y) u_{, y}+b_{1}(y) v \\
v_{, x} & a_{2}(y) v_{, y}+b_{2}(y) u
\end{array}\right], \quad \boldsymbol{E}(\boldsymbol{\phi})=\frac{1}{2}\left(\boldsymbol{G}+\boldsymbol{G}^{T}\right),
$$

where $a_{1}, a_{2}, b_{1}, b_{2}$ are Lipschitz continuous functions, such that $a_{1}(y)$ and $a_{2}(y)$ do not vanish on $[0, p]$. Then,

$$
\|\boldsymbol{G}\|^{2} \leq C\left(\frac{\|u\|\|\boldsymbol{E}\|}{h}+\|\boldsymbol{E}\|^{2}+\|\phi\|^{2}\right)
$$

where

$$
\|f\|^{2}=\int_{-h / 2}^{h / 2} \int_{0}^{p} f(x, y)^{2} d y d x .
$$

The proof is based on the sharp inequality for harmonic functions [3, Lemma 4.3], [7, Theorem 1.1] which we formulate here for the sake of completeness. 
LEMmA 5.2. Let $R_{h}=\left(-\frac{h}{2}, \frac{h}{2}\right) \times(0, p)$. Suppose $w \in H^{1}\left(R_{h}\right)$ is harmonic in $R_{h}$ and satisfies one of the conditions:

(i) $w(x, 0)=w(x, p)$ or in the sense of traces,

(ii) $w(x, 0)=0$ or in the sense of traces,

(iii) $w(x, p)=0$ or in the sense of traces.

Then

$$
\left\|w_{y}\right\|^{2} \leq \frac{2 \sqrt{3}}{h}\|w\|\left\|w_{x}\right\|+\left\|w_{x}\right\|^{2}
$$

Remark 5.3. For boundary conditions (3.8), the p-periodicity of the $u$ component of $\boldsymbol{\phi}$ in Theorem 5.1 must be replaced by either of the condition $v(x, 0)=0$ or $v(x, p)=0$ for all $x \in\left[-\frac{h}{2}, \frac{h}{2}\right]$. Then one must apply Lemma 4.1 to the extended version $\bar{\phi}$ of the displacement $\phi$ as follows:

$$
\begin{gathered}
u(x,-y)=u(x,-y), v(x, y)=-v(x,-y) \quad \text { for the condition } \quad v(x, 0)=0, \\
u(x, 2 p-y)=u(x,-y), v(x, y)=-v(x, 2 p-y) \quad \text { for the condition } \quad v(x, p)=0 .
\end{gathered}
$$

Then the extended displacement $\bar{\phi}$ satisfies the requirements of Theorem 5.1 .

Proof of Theorem 5.1. The first step is to replace $u(x, y)$ by its harmonic extension in $R_{h}$ by defining $w \in H^{1}\left(R_{h}\right)$ to be the unique solution of the Dirichlet boundary value problem

$$
\begin{cases}\triangle w=0, & (x, y) \in R_{h} \\ w=u, & (x, y) \in \partial R_{h}\end{cases}
$$

By the Poincaré inequality,

$$
\|u-w\| \leq h\|\nabla(u-w)\| .
$$

Next, we express $\triangle(u-w)=\Delta u$ in terms of $\boldsymbol{E}(x, y)$, defined in (5.1), by eliminating all derivatives, except $u_{, y}$ :

$$
\triangle(u-w)=E_{11, x}+\frac{2 E_{12, y}}{a_{1}}-\frac{1}{a_{1} a_{2}}\left(E_{22, x}+b_{2} E_{11}-b_{1} E_{22}\right)+R(x, y),
$$

where

$$
R(x, y)=\frac{b_{1} b_{2} u-a_{2} a_{1}^{\prime} u_{, y}-a_{2} b_{1}^{\prime} v}{a_{1} a_{2}} .
$$

Now we multiply (5.6) by $u-w$ and integrate by parts over $R_{h}$ using the fact that $u-w$ vanishes on $\partial R_{h}$ :

$$
\|\nabla(u-w)\|^{2}=E_{11}\left(u_{, x}-w_{, x}\right)+\frac{2 E_{12}\left(u_{, y}-w_{, y}\right)}{a_{1}}-\frac{E_{22}\left(u_{, x}-w_{, x}\right)}{a_{1} a_{2}}+(u-w) Q(x, y),
$$


where $Q(x, y)$ is a linear combination of $E_{11}, E_{12}, E_{22}, u, v$ and $u_{, y}$ with uniformly bounded coefficients. Estimating $\|u-w\|$ by (5.5) we obtain, after division by $\|\nabla(u-w)\|$,

$$
\|\nabla(u-w)\| \leq C h\left(\frac{\|\boldsymbol{E}\|}{h}+\|u\|+\|v\|+\left\|u_{, y}\right\|\right) .
$$

Our last task is to estimate $\left\|u_{, y}\right\|$. This is done by replacing $u$ with $w$, estimating $\left\|w_{, y}\right\|$ using (5.3), and returning back to $u$, while controlling the incurred errors by (5.5) and (5.7).

$$
\begin{aligned}
\left\|u_{, y}\right\|^{2} & \leq 2\left\|u_{, y}-w_{, y}\right\|^{2}+2\left\|w_{, y}\right\|^{2} \leq C\left(\|\nabla(u-w)\|^{2}+\frac{\|w\|\left\|w_{, x}\right\|}{h}+\left\|w_{, x}\right\|^{2}\right) \\
& \leq C\left(\|\nabla(u-w)\|^{2}+\left\|u_{, x}\right\|^{2}+\frac{1}{h}(\|u\|+h\|\nabla(u-w)\|)\left(\left\|u_{, x}\right\|+\|\nabla(u-w)\|\right)\right) \\
& \leq C\left(\|\nabla(u-w)\|^{2}+\left\|u_{, x}\right\|^{2}+\frac{\|u\|\left\|u u_{, x}\right\|}{h}+\frac{\|u\|\|\nabla(u-w)\|}{h}\right) \\
& \leq C\left(h^{2}\left\|u_{, y}\right\|^{2}+\|\boldsymbol{E}\|^{2}+\frac{\|u\|\|\boldsymbol{E}\|}{h}+\|u\|^{2}+\|v\|^{2}+\|u\|\left\|u_{, y}\right\|\right),
\end{aligned}
$$

where we took into account that $u_{, x}=E_{11}$. Estimating

$$
C\|u\|\left\|u u_{, y}\right\| \leq \frac{1}{2}\left\|u_{, y}\right\|^{2}+\frac{C^{2}\|u\|^{2}}{2}
$$

and choosing $h$ so small that $C h^{2}<1 / 4$ we obtain the inequality

$$
\|u, y\|^{2} \leq C\left(\|\boldsymbol{E}\|^{2}+\frac{\|u\|\|\boldsymbol{E}\|}{h}+\|u\|^{2}+\|v\|^{2}\right),
$$

which holds for all sufficiently small $h>0$. Observing that

$$
\|\boldsymbol{G}\|^{2} \leq\left\|E_{11}\right\|^{2}+\left\|E_{22}\right\|^{2}+\left\|G_{12}\right\|^{2}+\left\|2 E_{12}-G_{12}\right\|^{2},
$$

we get the bound

$$
\|\boldsymbol{G}\|^{2} \leq 7\|\boldsymbol{E}\|^{2}+3\left\|G_{12}\right\|^{2}
$$

while

$$
\left\|G_{12}\right\|^{2}=\left\|a_{1}(y) u_{, y}+b_{1}(y) v\right\|^{2} \leq C\left(\left\|u_{, y}\right\|^{2}+\|v\|^{2}\right) .
$$

This shows that (5.8) implies (5.2).

\section{Proof of Theorem 3.3}

The ansatz in part (i) of the theorem is a classical Kirchhoff ansatz. The assumptions of part (i) say that the shell contains a plate, which means that we can introduce a local Cartesian coordinate system $\left(x_{1}, x_{2}, x_{3}\right)$ in which the (sub)plate be described as

$$
\mathcal{P}_{h}=\left\{\left(x_{1}, x_{2}\right) \in \Omega \subset \mathbb{R}^{2}, x_{3} \in I_{h}\right\} .
$$


In these Cartesian coordinates we construct the ansatz in terms of the function $\phi\left(x_{1}, x_{2}\right)$, compactly supported in $\Omega$ :

$$
\left\{\begin{array}{l}
u_{1}^{h}=-x_{3} \phi_{, x_{1}}, \\
u_{2}^{h}=-x_{3} \phi_{, x_{2}}, \\
u_{3}^{h}=\phi\left(x_{1}, x_{2}\right) .
\end{array}\right.
$$

Then

$$
\nabla \boldsymbol{u}=\left[\begin{array}{ccc}
-x_{3} \phi_{, x_{1} x_{1}} & -x_{3} \phi_{, x_{1} x_{2}} & -\phi_{, x_{1}} \\
-x_{3} \phi_{, x_{1} x_{2}} & -x_{3} \phi_{, x_{2} x_{2}} & -\phi_{, x_{2}} \\
\phi_{, x_{1}} & \phi_{, x_{2}} & 0
\end{array}\right], \quad e(\boldsymbol{u})=\left[\begin{array}{ccc}
-x_{3} \phi_{, x_{1} x_{1}} & -x_{3} \phi_{, x_{1} x_{2}} & 0 \\
-x_{3} \phi_{, x_{1} x_{2}} & -x_{3} \phi_{, x_{2} x_{2}} & 0 \\
0 & 0 & 0
\end{array}\right] .
$$

This shows that

$$
\left\|\nabla \boldsymbol{u}^{h}\right\|^{2}=\|\nabla \phi\|^{2}+\frac{h^{2}}{12}\|\nabla \nabla \phi\|^{2}, \quad\left\|e\left(\boldsymbol{u}^{h}\right)\right\|^{2}=\frac{h^{2}}{12}\|\nabla \nabla \phi\|^{2} .
$$

Choosing fixed non-zero $\phi \in C_{0}^{2}(\Omega)$ we establish (3.6). The ansatz (6.1) was found by looking for the ansatz in the form $\boldsymbol{u}^{h}=\boldsymbol{v}\left(x_{1}, x_{2}\right)+x_{3} \boldsymbol{w}\left(x_{1}, x_{2}\right)$. We then compute $e\left(\boldsymbol{u}^{h}\right)=$ $\boldsymbol{E}_{0}\left(x_{1}, x_{2}\right)+x_{3} \boldsymbol{E}_{1}\left(x_{1}, x_{2}\right)$. The ansatz (6.1) is the general solution of the equations $\boldsymbol{E}_{0}\left(x_{1}, x_{2}\right)=$ 0 . The same idea could be applied to grad $\boldsymbol{u}$, given by (2.5). However, the different structure of the gradient results only in trivial solutions of $\boldsymbol{E}_{0}(\theta, z)=0$. Nevertheless we stick with the same idea, looking for an ansatz in the form $\boldsymbol{u}^{h}=\boldsymbol{v}^{h}(\theta, z)+t \boldsymbol{w}^{h}(\theta, z)$. Then,

$$
\left(\operatorname{grad} \boldsymbol{u}^{h}\right)_{\mathrm{sym}}=\boldsymbol{E}_{0}^{h}(\theta, z)+t \boldsymbol{E}_{1}^{h}(\theta, z)+O\left(t^{2}\right) .
$$

Now, instead of solving $\boldsymbol{E}_{0}^{h}(\theta, z)=0$ we demand that all components of $\boldsymbol{E}_{0}^{h}(\theta, z)$ be zero, except the zz-component. In accordance with this strategy we have the following system of equations

$$
\left\{\begin{array}{l}
w_{t}^{h}=0 \\
w_{\theta}^{h}=-\frac{1}{A_{\theta}}\left(\frac{\partial v_{t}^{h}}{\partial \theta}+c(\theta) v_{\theta}^{h}\right) \\
w_{z}^{h}=-\frac{1}{A_{z}} \frac{\partial v_{t}^{h}}{\partial z} \\
v_{t}^{h}=-\frac{v_{\theta, \theta}^{h}+a(\theta) v_{z}^{h}}{c(\theta)} \\
-A_{\theta} v_{\theta, z}^{h}=A_{z}\left(v_{z, \theta}^{h}-a(\theta) v_{\theta}^{h}\right)
\end{array}\right.
$$

The first four equations in (6.2) express $\boldsymbol{w}^{h}$ and $v_{t}^{h}$ in terms of $v_{\theta}^{h}$ and $v_{z}^{h}$. The last equation relates $v_{\theta}^{h}$ and $v_{z}^{h}$, and needs to be solved. There are two mutually exclusive cases

- Case 1:

$$
\frac{A_{z}}{A_{\theta}}=\frac{H(\theta)}{G(z)}
$$

for some Lipschitz functions $H(\theta)$ and $G(z)$. It is easy to see from formulas (2.4) that (6.3) is equivalent to $a(\theta)$ and $b(\theta)$ being linearly dependent, i.e. there exists a constant scalar $\lambda_{0}$, such that either $a(\theta)=\lambda_{0} b(\theta)$ or $b(\theta)=\lambda_{0} a(\theta)$. 
- Case 2: There exists an interval $I=\left(\theta_{1}, \theta_{2}\right) \subset(0, p)$, such that $a(\theta) \neq 0$ and $\rho^{\prime}(\theta) \neq 0$ for all $\theta \in I$, where

$$
\rho(\theta)=\frac{b(\theta)}{a(\theta)}
$$

Case 1. It is easy to see from Table 1 that all cylinders and cones fall into this case. Under the assumption (6.3) the last equation in (6.2) has a general solution

$$
v_{z}^{h}=A_{\theta} G(z) H(\theta) \phi_{, z}^{h}, \quad v_{\theta}^{h}=-A_{\theta} H(\theta)^{2} \phi_{, \theta}^{h},
$$

where $\phi^{h}(z, \theta)$ can be an arbitrary function with compact support.

Case 2. In this case we will assume that functions $a(\theta)$ and $b(\theta)$ are of class $C^{3}$. Solving the last equation in (6.2) with respect to $v_{z, \theta}^{h}$

$$
v_{z, \theta}^{h}=\frac{1}{B^{\prime}(z)}\left(a(\theta) B^{\prime}(z) v_{\theta}^{h}-(a(\theta) B(z)+b(\theta)) v_{\theta, z}^{h}\right)
$$

we see that we need both $a(\theta) v_{\theta}^{h}$ and $b(\theta) v_{\theta}^{h}$ to be $\theta$-derivatives of some $\theta$-periodic smooth functions of $(\theta, z)$. Hence, we define

$$
v_{\theta}^{h}=\frac{\psi_{, \theta}^{h}}{a(\theta)},
$$

where $\psi^{h}(\theta, z)$ is supported on $I \times\left(L_{-}, L_{+}\right)$. But then, we also need that $\rho(\theta) \psi_{, \theta}^{h}$ be a $\theta$-derivative of some $\theta$-periodic smooth function of $(\theta, z)$. We then define

$$
\psi^{h}=\frac{\phi_{, \theta}^{h}}{\rho^{\prime}(\theta)},
$$

where $\phi^{h}(\theta, z)$ is supported on $I \times\left(L_{-}, L_{+}\right)$. Then, we have

$$
\rho \psi_{, \theta}^{h}=\left(\rho \psi^{h}\right)_{, \theta}-\rho^{\prime} \psi^{h}=\left(\rho \psi^{h}-\phi^{h}\right)_{, \theta} .
$$

Hence,

$$
\left\{\begin{array}{l}
v_{\theta}^{h}=\frac{1}{a(\theta)} \frac{\partial}{\partial \theta}\left(\frac{\phi_{, \theta}^{h}}{\rho^{\prime}(\theta)}\right), \\
v_{z}^{h}=\frac{B^{\prime}(z) \phi_{, \theta}^{h}+\rho^{\prime}(\theta) \phi_{, z}^{h}-(B(z)+\rho(\theta)) \phi_{, \theta z}^{h}}{B^{\prime}(z) \rho^{\prime}(\theta)}
\end{array} .\right.
$$

Finally, in order to obtain optimal upper bound on the Korn constant we use the same scaling analysis as in [3] and define $\phi^{h}(\theta, z)$ in terms of the smooth, non-constant $p$-periodic in $\theta$ function $\Phi(\theta, z)$. In Case 1 we just set

$$
\phi^{h}(\theta, z)=\Phi(n(h) \theta, z),
$$

where $n(h)$ is the integer part of $h^{-1 / 4}$. In Case 2 we define

$$
\phi^{h}(\theta, z)=\eta(\theta, z) \Phi(n(h) \theta, z)
$$

where $\eta(\theta, z)$ is a smooth $p$-periodic in $\theta$ function, supported on $I \times\left(L_{-}, L_{+}\right)$. In both cases the constructed ansatz yields the upper bound $K\left(V_{h}\right) \leq C h^{3 / 2}$ for any $V_{h}$ containing $V_{h}^{1} \cap V_{h}^{2}$. 


\section{Acknowledgements.}

D.H. is grateful to Graeme Milton and the University of Utah for support. This material is based upon work supported by the National Science Foundation under Grants No. 1412058.

\section{References}

[1] P. G. Ciarlet. Mathematical elasticity. Vol. III, volume 29 of Studies in Mathematics and its Applications. North-Holland Publishing Co., Amsterdam, 2000. Theory of shells.

[2] D. Cioranescu, O. Oleinik, and G. Tronel. On Korn's inequalities for frame type structures and junctions. C. R. Acad. Sci. Paris Sér. I Math., 309(9):591-596, 1989.

[3] Y. Grabovsky and D. Harutyunyan. Exact scaling exponents in Korn and Korn-type inequalities for cylindrical shells. SIAM J. Math Anal., 46(5):3277-3295, 2014.

[4] Y. Grabovsky and D. Harutyunyan. Rigorous derivation of the buckling load in axially compressed circular cylindrical shells. J. Elasticity, 120(2):249-276, 2015. to appear.

[5] Y. Grabovsky and D. Harutyunyan. Scaling instability of the buckling load in axially compressed cylindrical shells. J. Nonlinear Sci., 26(1):83-119, 2016.

[6] Y. Grabovsky and L. Truskinovsky. The flip side of buckling. Cont. Mech. Thermodyn., 19(3-4):211-243, 2007.

[7] D. Harutyunyan. New asymptotically sharp Korn and Korn-like inequalities in thin domains. Journal of Elasticity, 117(1):95-109, October 2014.

[8] A. Korn. Solution générale du problème d'équilibre dans la théorie de l'élasticité, dans le cas ou les efforts sont donnés à la surface. In Annales de la faculté des sciences de Toulouse, volume 10, pages 165-269. Université Paul Sabatier, 1908.

[9] A. Korn. Über einige Ungleichungen, welche in der Theorie der elastischen und elektrischen Schwingungen eine Rolle spielen. Bull. Int. Cracovie Akademie Umiejet (Classe des Sci. Math. Nat.), pages 705-724, 1909.

[10] M. Lewicka and S. Muller. On the optimal constants in korn's and geometric rigidity estimates, in bounded and unbounded domains, under neumann boundary conditions. Indiana Math. Univ. Journal, to appear.

[11] A. E. H. Love. A treatise on the mathematical theory of elasticity. Dover, 4th edition, 1927.

[12] S. A. Nazarov. Weighted anisotropic Korn's inequality for a junction of a plate and a rod. Sbornik: Mathematics, 195(4):553-583, 2004.

[13] S. A. Nazarov. Korn inequalities for elastic junctions of massive bodies, thin plates, and rods. Russian Mathematical Surveys, 63(1):35, 2008. 
[14] O. A. Oleinik, A. S. Shamaev, and G. A. Yosifian. Mathematical problems in elasticity and homogenization, volume 26 of Studies in Mathematics and its Applications. NorthHolland Amsterdam, 1992.

[15] R. Paroni and G. Tomassetti. Asymptotically exact Korns constant for thin cylindrical domains. Comptes Rendus Mathematique, 350(15):749-752, 2012.

[16] R. Paroni and G. Tomassetti. On Korn's constant for thin cylindrical domains. Mathematics and Mechanics of Solids, 19(3):318-333, 2014.

[17] P. E. Tovstik and A. L. Smirnov. Asymptotic methods in the buckling theory of elastic shells, volume 4 of Series on stability, vibration and control of systems. World Scientific, 2001. 